

\title{
Efecto de Carragenatos, Proteína Aislada de Soya y Carmín sobre las Propiedades Fisicoquímicas y Sensoriales de la Pechuga de Pollo Cocida Estándar
}

\author{
Oscar García $^{1}$ (D), Rosmery Alvarado ${ }^{1}$, Iria Acevedo ${ }^{1}$ (D), Bruno Giménez López ${ }^{2}$ (D) \\ ${ }^{1}$ Laboratorio de Tecnología II. Procesos Agroindustriales. Programa de Ingeniería. Decanato de \\ Agronomía, Universidad Centroccidental Lisandro Alvarado. Barquisimeto, Venezuela. CP 3001. \\ ${ }^{2}$ Laboratorio de fisicoquímica, Facultad de Ingenierías, Escuela Profesional de Ingeniería \\ Agroindustrial, Universidad Privada San Juan Bautista, Ica, Perú. CP 11000. \\ *Autor de correspondencia: oscargarcia@ucla.edu.ve \\ https://doi.org/10.22209/rt.v44n3a03 \\ Recepción: 20 de enero de 2021 | Aceptación: 04 de junio de 2021 | Publicación: 01 de agosto 2021
}

\section{Resumen}

La carne de pollo es reconocida a nivel mundial por sus beneficios a la salud y su bajo costo comparada con la de cerdo, ternera y cordero. La presente investigación tuvo como objetivo determinar el nivel óptimo de carragenatos $(0,5 ; 1,0$ y $1,5 \%)$, proteína aislada de soya $(0,1$ y $2 \%)$ y carmín $(0 ; 0,1$ y $0,2 \%)$ para la elaboración de pechuga de pollo cocida estándar, generando un producto con aceptabilidad frente a marcas comerciales. Para ello, se establecieron 15 diferentes combinaciones según el diseño de superficie de respuesta de tipo Box-Behnken; el tamaño de cada unidad experimental fue de $400 \mathrm{~g}$ y se realizaron dos repeticiones por tratamiento, seguidamente a cada tratamiento, se le determinó la textura, color y actividad de agua. Los datos obtenidos se analizaron mediante un modelo lineal + interacción y se obtuvo la formulación óptima con una deseabilidad de 0,9239 . Luego, se caracterizó químicamente el producto, obteniéndose una humedad de 78,67 \%, proteína $15,18 \%$, grasa $0,55 \%$ y cenizas $2,78 \%$. Adicionalmente, la formulación optimizada se caracterizó sensorialmente mediante comparación con dos marcas comerciales, usando los parámetros de color, olor, sabor, textura y aceptación, con una escala estructurada de 5 puntos. El producto optimizado se presenta como una alternativa confiable para los consumidores y la industria procesamiento de pollo, debido a que presenta alto contenido de proteínas, bajo de grasas, rico en minerales y no presentó sinéresis.

Palabras clave: aceptabilidad; color; diseño Box-Behnken; textura.

\section{Effect of Carragenates, Soya Isolated Protein and Carmine on the Physicochemical and Sensory Properties of Standard Chicken Breast}

\begin{abstract}
The chicken meat is recognized worldwide for its health benefits and its low cost compared to pork, beef and lamb. The present investigation aimed to determine the optimal level of carrageenan $(0.5 ; 1$ and $1.5 \%)$, isolated soy protein $(0,1$ and $2 \%)$ and carmine $(0 ; 0.1$ and $0.2 \%)$ for the preparation of standard cooked chicken breast. For this, 15 different combinations were obtained according the Response Surface Design of the Box-Behnken, the size of each experimental unit was $400 \mathrm{~g}$ and two repetitions per treatment, after each treatment were evaluated the texture, color and water activity. The data obtained were analyzed through a linear model + interaction and the optimal formulation were achieved with a desirability of 0.9239 , then the product was characterized with chemical evaluations obtaining a humidity of $78.67 \%$, protein $15.18 \%$, fat $0.55 \%$ and ashes $2.78 \%$. Additionally the optimized product was performed sensory comparison with two commercial brands, evaluating the parameter color, smell, taste, texture and acceptance, with a structured scale of 5 points. The optimized product is a reliable
\end{abstract}


alternative for consumers and the chicken processing industry, due it has high protein content, low in fat, rich in minerals and did not show syneresis.

Keywords: acceptability; colour; design by Box-Behnken; texture.

\section{Introducción}

La carne de pollo es reconocida a nivel mundial por sus beneficios a la salud, entre los que están su alto valor nutricional, elevado contenido de proteínas, bajo contenido de colesterol, de calorías y grasas (Ayala, 2018). Por otro lado, en el ámbito económico la carne de pollo es menos costosa al compararla con otras carnes, como la de cerdo, ternera y cordero lo que en definitiva la hace más atractiva para el consumidor (Sujiwo et al., 2018). Esto ha traído como consecuencia que la producción de carne de aves de corral se incremente a nivel mundial y se prevé que sea el sector cárnico más grande con una producción aproximada de 130,7 millones de toneladas de carne para el año 2023 (Skarp et al., 2016). Por consiguiente, la carne proveniente de la pechuga de pollo es una gran fuente de proteínas a bajo costo, lo que proyecta un incremento en el consumo debido a una mayor conciencia saludable por parte de los consumidores. La pechuga de pollo cocida estándar se define como un producto elaborado con base de carne proveniente de la pechuga de aves destinado para el consumo humano, elaborado con sal, azúcar, especias y condimentos con la adición de gelificantes y proteína aislada de soya (COVENIN, 1997).

La textura está relacionada directamente con el contenido de agua y grasas presentes en los alimentos (Marín et al., 2006; Rodríguez et al., 2019). De esta manera, altos niveles de agua en la pechuga de pollo cocida influirán significativamente en la textura, siendo esto un factor determinante en el proceso de aceptación del producto por parte del cliente. Por ello, recomiendan disminuir al mínimo los niveles de agua durante el proceso de elaboración, de lo contrario se podría obtener un producto con características no deseadas (Attia et al., 2016). Por su parte, el aspecto externo apropiado debe ser consistente, liso, regular, sin grietas ni hundimientos ostensibles, pudiendo ir recubierta por una capa de gelificantes u otras materias primas autorizadas (Tuell et al., 2020).

Para obtener dichas características, la industria cárnica utiliza las carrageninas que son coloides que forman parte de los tejidos de las algas rojas y que poseen numerosas ventajas; como la capacidad de retención de agua, dando lugar a la formación de un gel que engloba moléculas de agua. Son agentes texturizante que imparten características funcionales específicas al producto final: mejoran el rebanado, proveen una consistencia homogénea con buena cohesión y disminuyen la sinéresis (Hee-Jin et al., 2020; Hongfang et al., 2021). En el mismo sentido, los aislados de soya se usan principalmente para mejorar la textura de los productos cárnicos, pero también para incrementar el contenido proteico, mejorar el sabor y como emulgente (Hongfang et al., 2021). La proteína aislada de soya contiene más de un $95 \%$ de proteína e interviene en el valor proteico (Palacios et al., 2010).

El color rojo de la carne se debe básicamente a la presencia de mioglobina, un pigmento muscular a quien se le atribuye la responsabilidad de proporcionar el color rojo característico de los embutidos. Sin embargo, la carne de pollo es baja en mioglobina, lo que hace que los embutidos elaborados a base de pollo tengan un color casi blanco, haciéndolos poco apetecibles por los consumidores. En tal sentido, el uso de carmín de cochinilla permite regular el color en la pechuga de pollo cocida estándar; este es un colorante natural soluble en agua con una tonalidad de tinción rosada, dependiendo del $\mathrm{pH}$, de la cantidad empleada, así como del producto a teñir, que presenta buena estabilidad al ser empleado en embutidos (Marroquín, 2009; Palacios et al., 2010).

El objetivo de la investigación fue determinar el nivel óptimo de carragenatos, proteína aislada de soya y carmín a utilizarse en la elaboración de pechuga de pollo cocida estándar, que genere un producto con aceptabilidad frente a marcas comerciales.

\section{Materiales y Métodos}

\section{Formulación, elaboración y análisis de propiedades fisicoquímicas del producto}

En el Tabla 1 se indican las diferentes combinaciones al utilizar los niveles de concentración de carragenatos $(0,5 ; 1,0$ y $1,5 \%)$ proteína aislada de soya $(0,1$ y $2 \%)$ y carmín $(0 ; 0,1$ y $0,2 \%)$, establecidas mediante un diseño de superficie de respuesta (DSR) del tipo Box-Behnken por el programa Minitab 17, para 3 factores 
independientes con 2 repeticiones para un total de 30 unidades experimentales. En cada tratamiento se midieron los indicadores físicos textura, color y actividad de agua (Aw).

Tabla 1. Combinaciones de los aditivos usadas en este trabajo, según el diseño de superficie de respuesta del tipo Box-Behnken, obtenidas en el programa Minitab 17.

\begin{tabular}{lrrrrr}
\hline Tratamiento & Bloque & Carragenatos (\%) & Proteína aislada de soya (\%) & Carmín (\%) \\
\hline 1 & 1 & 0,5 & 0 & 0,2 \\
2 & 1 & 1,0 & 2 & 0,1 \\
3 & 1 & 1,5 & 2 & 0,1 \\
4 & 1 & 0,5 & 1 & 0,0 \\
5 & 1 & 1,0 & 0 & 0,0 \\
6 & 1 & 1,5 & 1 & 0,2 \\
7 & 1 & 1,5 & 1 & 0,0 \\
8 & 1 & 1,5 & 0 & 0,2 \\
9 & 1 & 1,0 & 1 & 0,2 \\
10 & 1 & 0,5 & 1 & 0,1 \\
11 & 1 & 1,0 & 0 & 0,1 \\
12 & 1 & 1,0 & 1 & 0,1 \\
13 & 1 & 1,0 & 2 & 0,0 \\
14 & 1 & 1,0 & 1 & 0,2 \\
15 & 1 & 0,5 & 2 & 0,2 \\
16 & 2 & 0,5 & 0 & 0,2 \\
17 & 2 & 1,0 & 2 & 0,1 \\
18 & 2 & 1,5 & 2 & 0,1 \\
19 & 2 & 0,5 & 1 & 0,0 \\
20 & 2 & 1,0 & 0 & 0,0 \\
21 & 2 & 1,5 & 1 & 0,2 \\
22 & 2 & 1,5 & 1 & 0,0 \\
23 & 2 & 1,5 & 0 & 0,2 \\
24 & 2 & 1,0 & 1 & 0,2 \\
25 & 2 & 0,5 & 1 & 0,1 \\
26 & 2 & 1,0 & 0 & 0,1 \\
27 & 2 & 1,0 & 1 & 0,1 \\
28 & 2 & 1,0 & 2 & 0,0 \\
29 & 2 & 1,0 & 1 & 0,2 \\
30 & 2 & 0,5 & 2 & 0,2 \\
\hline & & & &
\end{tabular}

La pechuga de pollo se obtuvo en un frigorífico de la localidad, separándose la carne de los huesos de forma manual con la ayuda de cuchillos de acero inoxidable, para luego proceder a pesar la cantidad de carne según la formulación (aproximadamente $250 \mathrm{~g}$ ). La carne proveniente de la pechuga de pollo fue sometida a un proceso de molienda con discos de $3 \mathrm{~mm}$ de diámetro, manteniendo la temperatura por debajo de los $4{ }^{\circ} \mathrm{C}$, posteriormente se realizó el mezclado de la carne de pollo molida con salmuera, previamente preparada en una proporción de $67 \%$, conteniendo (\%): agua 27; fosfato 0,5 ; glutamato 0,5 ; azúcar 1 ; eritorbato 0,1 ; sal 1,5; sal de cura 0,2 ; carragenatos 0,6; aislado proteico de soya 1,6 y carmín 0,06. Con una mezcladora modelo Cuisinart, se realizaron 6 mezclados de 5 min a una velocidad de $180 \mathrm{rpm}$ y un reposo de $55 \mathrm{~min}$, para luego colocar la mezcla en refrigeración a $4{ }^{\circ} \mathrm{C}$ en un periodo de $24 \mathrm{~h}$, antes de proceder a embutir utilizando una funda sintética cero mermas, con una máquina embutidora manual. Una vez el embutido el producto, se cocinó a una temperatura de $95{ }^{\circ} \mathrm{C}$ por una $1 \mathrm{~h}$ en horno de convección, hasta obtener una temperatura interna de $71{ }^{\circ} \mathrm{C}$. Transcurrido el tiempo de cocción, se sumergió el producto en agua fría a $4{ }^{\circ} \mathrm{C}$ por $10 \mathrm{~min}$ y se almacenó en refrigeración a temperatura menores de10 ${ }^{\circ} \mathrm{C}$, hasta sus análisis.

En cuanto a la determinación de las propiedades fisicoquímicas del producto, en el caso de la dureza de la carne de pechuga, se midió utilizando un penetrómetro manual modelo Koehler. El color se determinó con un colorímetro Hunter Lab, calibrado con un azulejo blanco (calibración Minolta plato $\mathrm{N}^{\circ} 21733001, \mathrm{Y}=92,6 ; \mathrm{x}=$ 0,3136; $\mathrm{y}=0,3196)$ en $2^{\circ}$ ángulo de observación con una fuente iluminante $\mathrm{C}$ : Posteriormente, fueron seleccionadas 
dos lecturas por muestras de pechuga estándar. La luminosidad $\left(L^{*}\right)$ : blanco (100) hasta el negro (0), un enrojecimiento $\left(\mathrm{a}^{*}\right)$ : rojo $(+)$ hasta el verde $(-)$, amarillento $\left(\mathrm{b}^{*}\right)$ : amarillo $(+)$ hasta el azul $(-)$ y para la actividad de agua $(\mathrm{Aw})$, se usó un medidor Aqualab® $\mathrm{CX}-2$, directamente en la muestra de pechuga. Estos análisis se desarrollaron en el laboratorio de química agroindustrial de la UCLA.

\section{Análisis de regresión, gráficos de superficie y contorno, y deseabilidad}

Los análisis de regresión $\left(\mathrm{R}^{2}\right)$ son un criterio de valoración de la capacidad de explicación de los modelos de regresión, y representa el porcentaje de la varianza justificado por la variable independiente (Palacios et al., 2010). La Ecuación 1 representa en forma simbólica el modelo de respuesta, considerando todos los términos lineales cuadráticos y los términos de interacción lineal-lineal (Montgomery, 2004).

$$
Y=\beta_{0}+\sum \beta_{i} X_{i}+\sum \beta_{i i} X_{i i}^{2}+\sum \beta_{i j} X_{i} X_{j}+\epsilon
$$

Donde: $\beta_{0}$ es el término compensatorio; $\beta_{\mathrm{i}}$ es el término dependiente o el efecto lineal del factor de entrada $X_{\mathrm{i}}$; $\beta_{\mathrm{ii}}$ es el efecto cuadrático del factor de entrada $X_{\mathrm{i}}$ y $\beta_{\mathrm{ij}}$ es el efecto de interacción lineal-lineal entre el factor de entrada $X_{\mathrm{i}}$ y $X_{\mathrm{j}}$. La función de deseabilidad es la que permite obtener el punto factible donde los factores tienen un valor óptimo que equivale al grado de deseabilidad de la respuesta. Un valor de deseabilidad cercano a 1, quiere decir que la respuesta es deseable.

\section{Análisis fisicoquímico del producto terminado}

Se determinaron los siguientes parámetros: humedad (COVENIN 1077-97, 1997), proteínas (COVENIN 1218-80, 1980), grasas (COVENIN 3218-96, 1996) y cenizas (COVENIN 1220-1999, 1999).

\section{Evaluación de la aceptabilidad del producto}

En esta fase se empleó una prueba afectiva con un panel sensorial no entrenado de 70 evaluadores, con una escala estructurada de 5 puntos, conformado por personas del sector San José del municipio Iribarren del estado Lara (Venezuela). Los factores evaluados fueron: sabor, olor, color, textura y aceptación, para niveles de "desagrado $=1$ " hasta "me gusta mucho $=5$ ". A cada persona se le suministró una muestra del mejor tratamiento, así como de las 2 marcas comerciales usadas de referencia, las cuales se denominaron Mc1 y Mc2, con códigos en orden aleatorio.

\section{Análisis estadístico}

Se usó el programa Minitab 17 para el análisis de los resultados de las variables físicas y químicas, con la finalidad de obtener el producto optimizado, y se utilizaron las pruebas no paramétricas de Kruskal-Wallis y la prueba de la mediana de Mood, para demostrar la significancia $p<0,05$ en el análisis sensorial.

\section{Resultados y Discusión}

\section{Fase 1: formulación de niveles de carragenatos, proteína aislada de soya y carmín}

\section{Determinación de las propiedades físicas}

En la Tabla 2 se muestran los 15 tratamientos en estudio de acuerdo al diseño de superficie de respuesta (DOE) tipo Box Behnken, obtenido en el programa Minitab 17, con los respectivos resultados de las variables estudiadas. 
Tabla 2. Valores obtenidos experimentalmente de textura, actividad de agua (Aw) y color en las diferentes formulaciones de pechuga de pollo estándar utilizadas según el diseño de superficie de respuesta del tipo BoxBehnken.

\begin{tabular}{ccccccccc}
\hline \multirow{2}{*}{ Tratamiento } & Carragenatos & \multirow{2}{*}{$\begin{array}{c}\text { Proteína } \\
\text { de soya }\end{array}$} & Carmín & \multicolumn{3}{c}{ Color } & \multirow{2}{*}{$\begin{array}{c}\text { Textura } \\
(\mathrm{mm})\end{array}$} & Aw \\
\cline { 6 - 7 } 1 & 2,76 & 0,00 & 1,1 & 49,52 & 27,41 & 06,81 & 169 & 0,971 \\
2 & 5,52 & 11,04 & 0,5 & 61,22 & 12,74 & 13,03 & 197 & 0,975 \\
3 & 8,28 & 11,04 & 0,5 & 61,54 & 14,64 & 14,31 & 200 & 0,970 \\
4 & 2,76 & 5,52 & 0,0 & 69,15 & 05,92 & 17,28 & 191 & 0,970 \\
5 & 5,52 & 0,00 & 0,0 & 72,55 & 06,13 & 16,25 & 186 & 0,974 \\
6 & 8,28 & 5,52 & 1,1 & 58,77 & 16,08 & 13,05 & 190 & 0,970 \\
7 & 8,28 & 5,52 & 0,0 & 63,67 & 06,59 & 17,98 & 190 & 0,973 \\
8 & 8,28 & 0,00 & 1,1 & 62,06 & 20,51 & 09,55 & 168 & 0,970 \\
9 & 5,52 & 5,52 & 1,1 & 62,82 & 17,85 & 11,45 & 171 & 0,971 \\
10 & 2,76 & 5,52 & 0,5 & 65,17 & 11,36 & 14,48 & 172 & 0,970 \\
11 & 5,52 & 0,00 & 0,5 & 69,73 & 10,35 & 14,75 & 172 & 0,970 \\
12 & 5,52 & 5,52 & 0,5 & 67,52 & 10,28 & 15,10 & 183 & 0,970 \\
13 & 5,52 & 11,04 & 0 & 68,69 & 6,58 & 18,88 & 174 & 0,970 \\
14 & 5,52 & 5,52 & 1,1 & 60,22 & 19,49 & 10,84 & 176 & 0,970 \\
15 & 2,76 & 11,04 & 1,1 & 61,87 & 17,73 & 13,03 & 198 & 0,970 \\
\hline
\end{tabular}

L*: luminosidad; $\mathrm{a}^{*}$ : enrojecimiento; $\mathrm{b}^{*}$ : amarillento.

En la Tabla 3 se presentan los valores de los coeficientes de regresión $\left(\mathrm{R}^{2}\right)$ estimados de la luminosidad $\left(\mathrm{L}^{*}\right)$, enrojecimiento $\left(\mathrm{a}^{*}\right)$, amarillento $\left(\mathrm{b}^{*}\right)$, textura y Aw, obtenidos a partir de tres modelos poblacionales de respuesta de predicción. El primero es un modelo lineal en el cual se puede observar que no hubo significancia, debido a que sus valores de $\mathrm{R}^{2}$ fueron inferiores al $80 \%$ para $\mathrm{L}^{*}$, textura y $\mathrm{Aw}$, mientras que fueron levemente superiores a $80 \%$ para a* y b*. Para el segundo modelo basado en la respuesta de una función lineal + cuadrática, los valores de $\mathrm{R}^{2}$ tampoco fueron significativos obteniéndose resultados muy parecidos a los encontrados en el primer modelo. Sin embargo, al utilizarse el tercer modelo lineal + interacción los coeficientes de $\mathrm{R}^{2}$ indicaron una buena capacidad de predicción, con resultados cercanos o superiores al $80 \%$ para $\mathrm{L}^{*}, \mathrm{a}^{*}, \mathrm{~b}^{*}$ y la textura, mientras que para $\mathrm{Aw}$ el $\mathrm{R}^{2}$ fue significativamente inferior al $80 \%$. Por lo tanto, como los coeficientes de determinación para Aw fueron inferiores al $80 \%$ para las tres funciones, no será considerado para decidir cuál es formula de optimización de la pechuga de pollo estándar.

Tabla 3. Coeficientes de determinación $\left(\mathrm{R}^{2}\right)$ de cada modelo estadístico de color, textura y actividad de agua.

\begin{tabular}{cccccc}
\hline & \multicolumn{5}{c}{$\mathrm{R}^{2}(\%)$} \\
\cline { 2 - 6 } Resumen del modelo & $\mathrm{L}^{*}$ & $\mathrm{a}^{*}$ & $\mathrm{~b}^{*}$ & Textura & $\mathrm{Aw}$ \\
\hline Lineal & 50,99 & 83,94 & 83,47 & 40,62 & 15,09 \\
Lineal + cuadrado & 61,59 & 83,94 & 83,47 & 43,49 & 13,07 \\
Lineal + interacción & 81,02 & 94,81 & 89,96 & 70,20 & 40,44 \\
\hline
\end{tabular}

$\mathrm{L}^{*}$ : luminosidad, $\mathrm{a}^{*}$ : enrojecimiento, $\mathrm{b}^{*}$ : amarillento, Aw: actividad de agua.

Al analizar las varianzas se refleja que las variables respuestas para $\mathrm{L}^{*}, \mathrm{a}^{*}, \mathrm{~b}^{*}$ y textura presentaron diferencias estadísticamente significativas $(p<0,05)$, con un nivel de confianza del $95 \%$, tal como se observa en la Tabla 4. 
Tabla 4. Coeficientes de regresión de luminosidad $\left(\mathrm{L}^{*}\right)$, enrojecimiento ( $\left.\mathrm{a}^{*}\right)$, amarillento ( $\mathrm{b}^{*}$ ) y textura para un modelo lineal + interacciones + cuadrados con mejores predicciones de $\mathrm{R}^{2}$.

\begin{tabular}{lccccc}
\hline Parámetros & Factor & L* & $\mathrm{a}^{*}$ & $\mathrm{~b} *$ & Textura \\
\hline Constante, & $\mathrm{b}_{0}$ & $59,12^{* *}$ & $13,19 * *$ & 13,63 & $223 * * *$ \\
Carragenatos & $\mathrm{b}_{1}$ & 4,60 & $-2,16$ & $0,68 \mathrm{~s}$ & $-11,62 * *$ \\
Proteína de soya & $\mathrm{b}_{2}$ & 0,72 & $-1,24$ & $0,77 * *$ & $-3,78 * * *$ \\
Carmín & $\mathrm{b}_{3}$ & $-15,67 * * *$ & $15,72 * * *$ & $-8,27 * * *$ & $-36,90$ \\
Carragenatos $\times$ carragenatos & $\mathrm{b}_{1}^{*} \mathrm{~b}_{1}$ & $-0,38^{* *}$ & 0,14 & $-0,02$ & 0,94 \\
Proteína soya $\times$ proteína soya & $\mathrm{b}_{2}^{*} \mathrm{~b}_{2}$ & 0,0004 & $0,0585^{* * *}$ & $-0,0308^{*}$ & 0,13 \\
Carmín $\times$ carmín & $\mathrm{b}_{3}^{*} \mathrm{~b}_{3}$ & $-4,35$ & 3,15 & 0,43 & 2,70 \\
Carragenatos $\times$ proteína de soya & $\mathrm{b}_{1} * \mathrm{~b}_{2}$ & $-0,23 * * *$ & $0,15 * * *$ & $-0,07 * *$ & $0,29 * *$ \\
Carragenatos $\times$ carmín & $\mathrm{b}_{1} * \mathrm{~b}_{3}$ & $1,40 * *$ & $-0,70 * *$ & 0,09 & 1,50 \\
Proteína de soya $\times$ carmín & $\mathrm{b}_{2} * \mathrm{~b}_{3}$ & $0,77 * *$ & $-0,58 * * *$ & 0,22 & $4,04 * *$ \\
\hline
\end{tabular}
$* * * p \leq 0,01 ; * * 0,01 \leq p \leq 0,05$.

\section{Análisis gráfico de las superficies de respuestas de las variables textura y color}

En la Figura 1 se puede observar que en ausencia de proteína aislada de soya, la textura fue baja, mientras que a medida que aumentaba la cantidad de proteína aislada de soya, los niveles de textura fueron superiores a 180 $\mathrm{mm}$, registrándose un pico correspondiente a la mayor cantidad de carragenatos de proteína aislada de soya, donde se observó, además, la mayor dureza de textura en el producto. El uso de estos extensores cárnicos permite trabajar con un amplio rango de texturas, porque estos agentes texturizante imparten características funcionales específicas al producto final (Rodríguez et al., 2015).

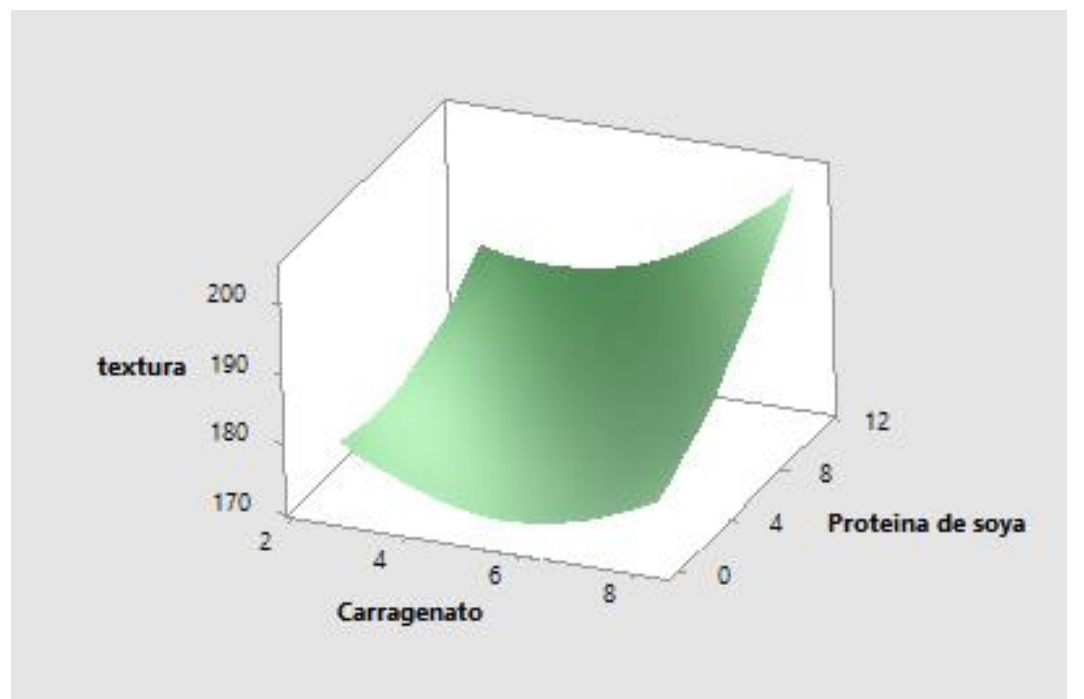

Figura 1. Superficie de textura vs. proteína de soya, carragenatos de pechuga de pollo estándar.

Como se observa en la Figura 2, con altas concentraciones de carragenatos, proteína aislada de soya y carmín, se obtiene una disminución significativa de la $\mathrm{L}^{*}$ del producto elaborado, esto es debido a que hay un aumento en la captación de agua por parte del gel formado por las proteínas y los carragenatos.

En la Figura 2 se observa que a medida que aumenta la concentración de proteína aislada de soya y de carragenatos, el a* del producto disminuyo significativamente, mientras que al aumentar la concentración de carmín aumento el nivel de enrojeciendo del producto. Esto puede ser debido a que el carmín imparte una tinción rosada a los embutidos y la tonalidad depende de la cantidad empleada (Tinoco et al., 2011). Para el caso específico de la 
presencia de carmín, en la Figura 2, se puede observar que el b* disminuye a medida que aumenta la concentración de carmín.

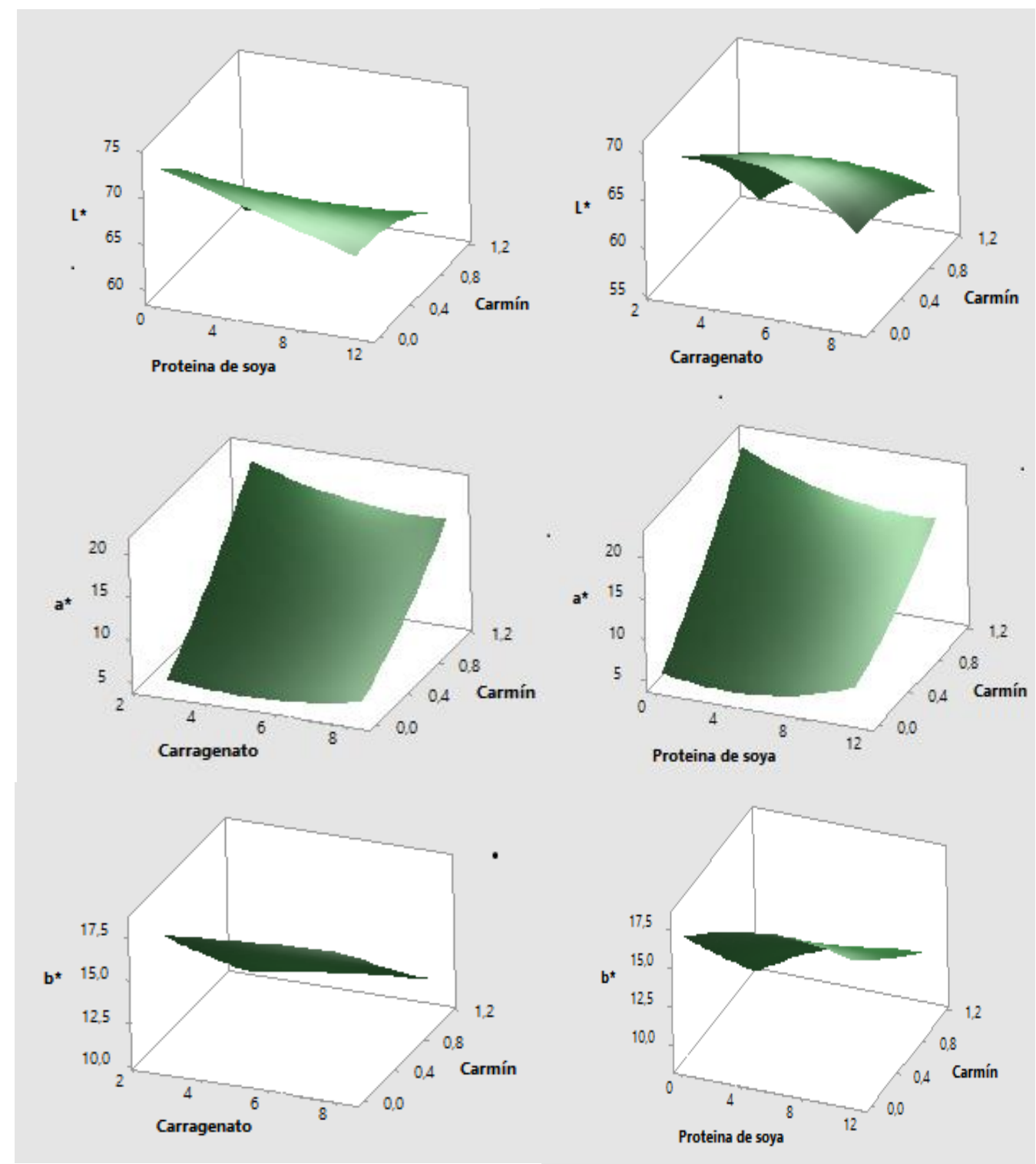

Figura 2. Superficie de respuesta de luminosidad $\left(\mathrm{L}^{*}\right)$, enrojecimiento $\left(\mathrm{a}^{*}\right)$ y amarillento $\left(\mathrm{b}^{*}\right)$ vs. carmín, proteína de soya; carmín, carragenatos

La Figura 3, ilustra un resumen de los resultados de la función de deseabilidad obtenida. Las columnas presentan los tres factores y en las filas las variables de textura y color $\left(\mathrm{L}^{*}, \mathrm{a}^{*} \mathrm{y} \mathrm{b}^{*}\right)$. La respuesta obtenida fue una deseabilidad D: 0,924, por lo tanto, el proceso alcanzó una respuesta óptima global con 3,277 g de carragenatos $(0,6$ $\%), 8,852$ g proteína aislada de soya $(1,6 \%)$ y $0,285 \mathrm{~g}$ de carmín $(0,06 \%)$. Los objetivos de las variables dependientes fueron obtenidos por productos comerciales. 


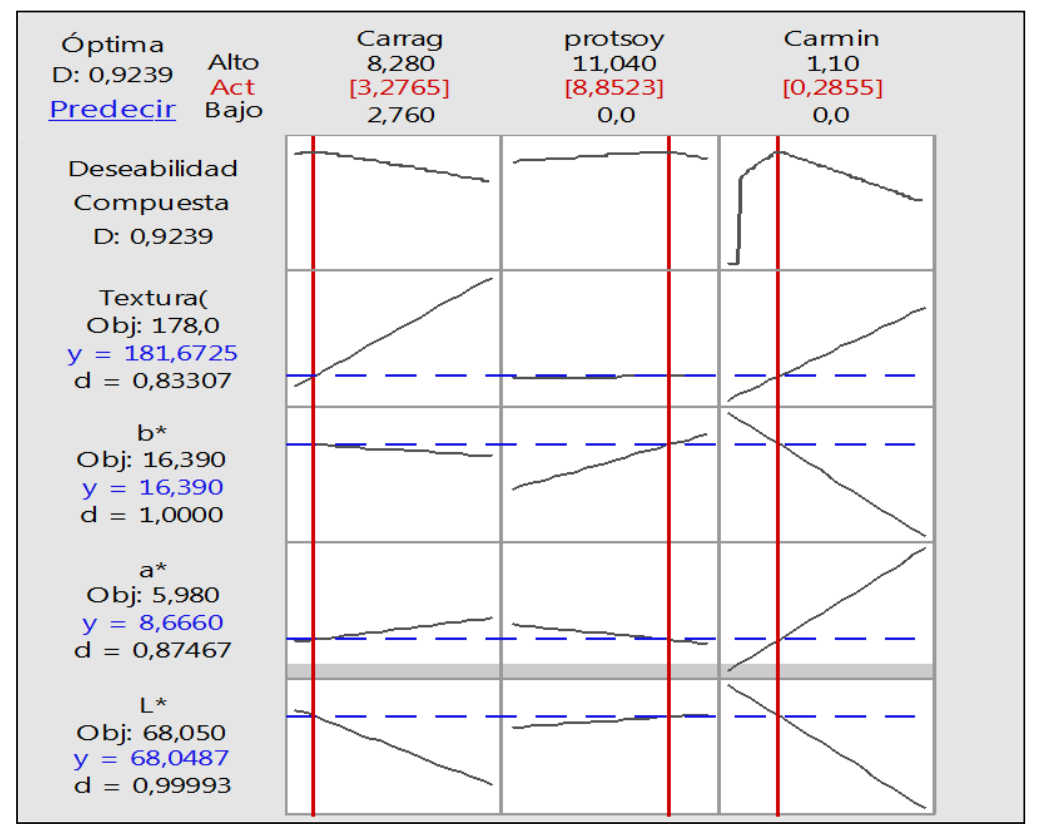

Figura 3. Resumen óptimo de la deseabilidad en la pechuga estándar. D: deseabilidad, Carrag: carragenatos, protsoy: proteína de soya, $\mathrm{L}^{*}$ : luminosidad, $\mathrm{a}^{*}$ : enrojecimiento, $\mathrm{b}^{*}$ : amarillento.

\section{Fase 2: determinación fisicoquímica del producto optimizado}

La caracterización química, tal como se observa en la Tabla 5, mostró un porcentaje de humedad en el producto óptimo de pechuga de pollo cocida estándar de 78,67\%, encontrándose en la tabla nutricional de productos comerciales de pechuga de pollo cocida estándar valores inferiores a 75,80 \% (Hongfang et al., 2021). El porcentaje de humedad encontrado se debe al uso de los extensores cárnicos, tanto de carrageninas y proteína aislada de soya, ya que poseen gran capacidad de retención de agua porque forman redes tridimensionales, dando lugar a la formación de un gel, haciendo el producto final más jugoso, ya que absorbe agua (Keenan et al., 2014).

El porcentaje de proteína en el producto de pechuga de pollo cocida estándar fue de $15,17 \%$, el cual se encuentra dentro de los requisitos químicos establecidos por COVENIN 3305:1997 (1997), que indica un mínimo de $15 \%$ de proteína de origen animal. Una de las propiedades que diferencian la carragenina de otros hidrocoloides es su habilidad de interactuar con las proteínas de los alimentos a través de puentes entre el éster sulfato de la carragenina con los residuos carboxílicos de los aminoácidos (Marroquín, 2009; Janacua, 2016).

En cuanto al porcentaje de grasa en el producto óptimo, el mismo fue de $0,548 \%$ en $100 \mathrm{~g}$ de muestra, y se encontró que en la tabla nutricional de productos comerciales de pechuga de pollo cocida estándar, los porcentajes de grasa son de 1 a $2 \%$. A medida que se sustituye proteína de soya por carne, el porcentaje de grasa disminuye, es decir, el extensor cárnico ayuda a obtener un producto más saludable (Marroquín, 2009; Palacios et al., 2010).

La pechuga de pollo optimizada presentó un porcentaje de cenizas de $2,78 \%$, siendo este un nivel alto ,comparado con el reportado por Attia et al. (2016) quienes obtuvieron un 1\% de cenizas para la pechuga de pollo sin tratamientos. Este incremento en el porcentaje de cenizas puede ser atribuido a la presencia de los carragenatos en el producto cárnico, debido a que estos tiene presente en su molécula átomos de hierro, calcio, fósforo, magnesio y molibdeno quienes son los contribuyen en la cantidad de minerales presentes en las cenizas (Palacios et al., 2010; Rodrígues et al., 2019).

Tabla 5. Caracterización fisicoquímica de la pechuga estándar optimizada.

\begin{tabular}{lcccc}
\hline \multicolumn{1}{c}{ Muestra } & Humedad (\%) & Proteína (\%) & Grasas (\%) & Cenizas (\%) \\
\hline COVENIN 3305-97 & - & Mín. 15 & Máx. 2 & - \\
Pechuga estándar & 78,67 & 15,17 & 0,548 & 2,782 \\
\hline
\end{tabular}




\section{Fase 3: evaluación de la aceptabilidad}

La prueba de Kruskal-Wallis reflejó que las medianas de los tratamientos fueron diferentes, lo que indica que hubo diferencia significativa entre las marcas comerciales y el tratamiento óptimo $(p<0,05)$. Lo mismo se encontró al analizar los resultados mediante la prueba de mediana de Mood. El producto optimizado resultó superior en todos los atributos evaluados con respecto a los comerciales. En la Tabla 6, se observan los valores obtenidos de las pruebas no paramétricas para color, olor, apariencia, textura y sabor.

Tabla 6. Resultados de las pruebas no paramétricas color, olor, apariencia, textura y sabor de la pechuga estándar optimizada con respecto a dos marcas comerciales.

\begin{tabular}{|c|c|c|c|c|c|c|}
\hline \multirow[t]{2}{*}{ Atributo } & \multirow[t]{2}{*}{ Muestra } & \multicolumn{3}{|c|}{ Prueba de Kruskal-Wallis } & \multicolumn{2}{|c|}{$\begin{array}{c}\text { Prueba de mediana de } \\
\text { Mood }\end{array}$} \\
\hline & & Mediana & H-P & & Mediana & $\mathrm{P}$ \\
\hline \multirow[t]{3}{*}{ Color } & Mc1 & 4,000 & $\mathrm{H}=18,73 \mathrm{GL}=2$ & $\mathrm{P}=0,000$ & 4,000 & \\
\hline & $\mathrm{Mc} 2$ & 4,000 & $\mathrm{H}=21,78 \mathrm{GL}=2$ & $P=0,000$ & 4,000 & 0,002 \\
\hline & Trat opt & 5,000 & & & 5,000 & \\
\hline \multirow[t]{3}{*}{ Olor } & Mc1 & 4,000 & $\mathrm{H}=11,00 \mathrm{GL}=2$ & $\mathrm{P}=0,004$ & 4,000 & 0,012 \\
\hline & $\mathrm{Mc} 2$ & 4,000 & $\mathrm{H}=13,21 \mathrm{GL}=2$ & & 4,000 & \\
\hline & Trat opt & 5,000 & $P=0,001$ & & 5,000 & \\
\hline \multirow{3}{*}{ Apariencia } & 1 & 4,000 & $\mathrm{H}=18,06 \mathrm{GL}=2$ & $\mathrm{P}=0,000$ & 4,000 & 0,000 \\
\hline & Mc2 & 4,000 & $\mathrm{H}=22,68 \mathrm{GL}=2$ & $\mathrm{P}=0,000$ & 4,000 & \\
\hline & Trat opt & 5,000 & & & 5,000 & \\
\hline \multirow[t]{3}{*}{ Textura } & $\mathrm{Mc1}$ & 4,000 & $\mathrm{H}=15,14 \mathrm{GL}=2$ & $\mathrm{P}=0,001$ & 4,000 & 0,000 \\
\hline & Mc2 & 4,000 & $\mathrm{H}=18,45 \mathrm{GL}=2$ & $P=0,000$ & 4,000 & \\
\hline & Trat opt & 5,000 & & & 5,000 & \\
\hline \multirow[t]{3}{*}{ Sabor } & Mc1 & 4,000 & $\mathrm{H}=6,22 \mathrm{GL}=2$ & $\mathrm{P}=0,041$ & 4,000 & 0,0419 \\
\hline & $\mathrm{Mc} 2$ & 4,000 & $\mathrm{H}=7,59 \mathrm{GL}=2$ & $\mathrm{P}=0,022$ & 4,000 & \\
\hline & Trat opt & 5,000 & & & 5,000 & \\
\hline
\end{tabular}

Mc1: marca comercial 1, Mc2: marca comercial 2, Trat opt: tratamiento óptimo, H-P: prueba estadística de Kruskall Wallis.

Finalmente, se puede proponer la metodología de Box-Behnken como técnica de para la formulación de nuevos productos en la industria de los productos procesados de la carne.

\section{Conclusiones}

La formulación óptima de la pechuga estándar es de 0,6\% de carragenatos, 1,6 \% de proteína de aislado de soya y $0,06 \%$ de carmín con una deseabilidad de $0,9239 \%$. Por lo que el tratamiento óptimo es bajo en grasa, rico en minerales, contiene un alto nivel proteico, y además, posee una buena fuerza de cohesión y alta capacidad de retención de agua. En la evaluación sensorial, el tratamiento óptimo con respecto a las dos marcas comerciales, presentó valores significativamente superiores $(p<0,05)$ en cuanto a color, olor, apariencia, textura y sabor. La formulación determinada en esta investigación, permite mejorar la calidad nutricional y organoléptica de la pechuga de pollo cocida estándar, debido a que se demostró que es aceptable por un panel de consumidores.

\section{Referencias Bibliográficas}

Attia, Y., Al, M., Korish, M., Shiboob, M. (2016). Evaluación de la calidad de la carne de pollo en el mercado minorista: efectos del tipo y origen de las canales. Revista Méexicana de Ciencias Pecuarias, 7(3), 321-339.

Ayala, C. (2018). Importancia nutricional de la carne. Revista de Investigación e Innovación Agropecuaria y de Recursos Naturales, 5(especial), 54-61.

COVENIN 3305-97. (1997). Pechuga de pollo. Caracas-Venezuela. Comisión Venezolana de Normas Industriales 
(COVENIN), Publicaciones Fondonorma.

Hee-Jin, K., Hye-Jin, K., JinJoo, J., Ki-Chang, N., Kwan-Seob, S., Jong-Hyun, J., Kyong, S., Yangil, C., Sang-Ho, K., Aera, J. (2020). Comparison of the quality characteristics of chicken breast meat from conventional and animal welfare farms under refrigerated storage. Poultry Science, 99(3), 1788-1796.

Hongfang, J., Xianzhe, H, Lingwen, Z., Xuefei, W., Hanjun, M., Fusheng, C. (2021). Effect of ice-temperature storage on someproperties of salt-soluble proteins and gel fromchicken breast muscles. Journal of Food, 19(1), 521531.

Marín, E., Lemus, R., Flores, V., Vega, A. (2006). La rehidratación de los alimentos deshidratados. Revista Chilena de Nutrición, 33(3), 527-538.

Marroquín, J. (2009). Influencia del chocho (lupinus mutabilis) y carragenina en combinación con carne de pollo en la elaboración de carne para hamburguesa. Tesis de grado. Ibarra: Universidad Técnica del Norte.

Montgomery, D. C. (2004). Diseño y analisis de experimentos. $2^{\text {da }}$ ed. México: Editorial Limusa, S. A.

Palacios, A., Loyola, W. (2010). Elaboración de chorizo y salchicha frankfurt a partir de proteína de soya (Glycine max). Tesis de grado. Cuenca: Universidad Politécnica Salesiana, Ecuador.

Rodríguez, J., Tolotti, F., Levandowski, R. (2019). Mortadella formulations using poultry breasts with White Striping and Wooden Breast. Revista Brasileira de Higiene e Sanidade Animal, 13(3), 411-417.

Rodríguez, D., Giraldo, E., Restrepo, D. (2015). Evaluación sensorial e instrumental de textura de salchichones tipo estándar que contienen un extensor cárnico de pasta de pollo. Revista de la Facultad de Ciencias Farmacéuticas y Alimentarias, 22(2), 2145-2660.

Skarp, C., Hänninen, M., Rautelin, H. (2016). Campylobacteriosis: the role of poultry meat. Clinical Microbiology Infection, 22(2), 103-109.

Sujiwo, J., Dongwook, K., Jang, A. (2018). Relation among quality traits of chicken breast meat during cold storage: correlations between freshness traits and torrymeter values. Poultry Science, 97, 887-2894.

Tinoco, M., Rojas, C., Tripaldi, P. (2011). Aplicación de funciones de decisión multicriterio y diseño PlackettBurman para el estudio de la calidad sensorial de mortadelas. Revista Venezolana de Ciencia y Tecnología de Alimentos, 2(1), 142-157.

Tuell, J., Jun-Young, P., Weichao, W., Heng-Wei, C., Yuan, B., Kim, T. (2020). Functional physicochemical properties and oxidative stability of ground meat from broilers reared under different photoperiods. Poultry Science, 99, 3761-3768.

Keenan, D., Resconi, V., Kerry, J., Hamill, R. (2014). Modelling the influence of inulin as a fat substitute in comminuted meat products on their physico-chemical characteristics and eating quality using a mixture design approach. Meat Science, 96(3), 1384-1394. 


\section{REVISTA TECNICA}

DE LA FACULTAD DE INGENIERIA

UNIVERSIDAD DEL ZULIA

Vol. 44. $\mathbf{N}^{\circ} 3$, Septiembre - Diciembre, 2021

Esta revista fue editada en formato digital y publicada en Agosto 2021, por el Fondo Editorial Serbiluz, Universidad del Zulia. Maracaibo-Venezuela

www.luz.edu.ve

www.serbi.luz.edu.ve

www.produccioncientificaluz.org 